\title{
Misdiagnosis of primary pleural DLBCL as tuberculosis: A case report and literature review
}

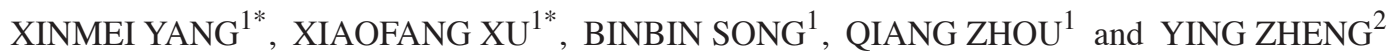 \\ ${ }^{1}$ Department of Oncology and ${ }^{2}$ The Central Laboratory, The First Hospital of Jiaxing, Jiaxing, Zhejiang 314001, P.R. China
}

Received November 15, 2017; Accepted March 27, 2018

DOI: $10.3892 / \mathrm{mco} .2018 .1601$

\begin{abstract}
Diffuse large B-cell lymphoma (DLBCL) is the most common type of non-Hodgkin lymphoma (NHL). DLBCL presents with pleural involvement at an advanced stage; however, primary pleural lymphomas without any other site of involvement are rare, and the possibility of misdiagnosis is high, particularly in developing countries, where tuberculosis or other severe pulmonary infections remain a major health concern. Furthermore, lymphoma and tuberculosis share a number of common clinical characteristics, such as fever, night sweats, feeling of satiety after a small meal, fatigue and unexplained weight loss, among others. We herein describe a case of misdiagnosis of primary pleural lymphoma as tuberculosis in a 49-year-old male patient who presented with pleural effusion and high adenosine deaminase (ADA) level in the pleural fluid. Anti-tuberculosis treatment was administered for 1 month, but the patient's condition deteriorated. A surgical biopsy was performed and was diagnostic of DLBCL. CHOP chemotherapy was administered with a significant delay due to the misdiagnosis, and it was not efficient, as rituximab was not added to the regimen. The therapeutic efficacy was monitored by computed tomography scans, which revealed that the lesion had shrunk slightly. The overall survival of the patient was $\sim 1$ year and he eventually succumbed to severe thoracic infection and pleural effusion. Suspicion should be raised when a patient presents with pleural effusion and extremely high ADA levels, as ADA activity of $>250 \mathrm{U} / \mathrm{L}$ should raise the suspicion of empyema or lymphoma rather than tuberculosis.
\end{abstract}

\section{Introduction}

Pleural involvement in patients with non-Hodgkin lymphoma (NHL) is well documented, and $20 \%$ of the cases present with

Correspondence to: Dr Ying Zheng, The Central Laboratory, The First Hospital of Jiaxing, 1882 Zhonghuan South Road, Jiaxing, Zhejiang 314001, P.R. China

E-mail: jiaxinghospital@163.com

${ }^{*}$ Contributed equally

Key words: non-Hodgkin lymphoma, diffuse large B-cell lymphoma, pleural effusion, tuberculosis, tuberculous pleurisy pleural effusion (1-3). However, primary pleural lymphomas without any other site of involvement are extremely rare, accounting for $\sim 0.3 \%$ of all NHLs. Malignant lymphoma of the pleura has been mostly associated with chronic pleural inflammation (4). Therefore, the treating physicians must include primary pleural NHL in the differential diagnosis when a patient presents with pleural inflammation or effusion, particularly in China, where tuberculosis (TB) and severe lung infections remain a major public health concern. The case reported herein is of particular interest, as the patient had no history of pleuro-pulmonary disease or evidence of lymphoma at any other site.

\section{Case report}

A 49-year-old man presented to the outpatient care of the First Hospital of Jiaxing (Jiaxing, China) on May 12, 2014 with a lump on the left chest wall accompanied by pain. A chest computed tomography (CT) scan suggested possible tuberculous pleurisy along with left pulmonary TB. In addition, bone destruction was observed in some ribs on the left side, along with the formation of a cold abscess. Single-photon emission computed tomography revealed particularly high radioactive ${ }^{99 \mathrm{~m}} \mathrm{Tc}$ uptake in the left anterior thoracic wall. The ratio of target (area of highest uptake) to non-target (normal tissue) was 117.72. The fiberoptic bronchoscopic and hydrothorax exfoliative cytological examinations were negative for malignant cells. Biochemical analysis of the pleural fluid revealed a markedly high adenosine deaminase (ADA) level (401.2 IU/L). The differential white blood cell count revealed $98 \%$ lymphocytes and $2 \%$ neutrophils. The fluid was brown in color and cloudy. The fluid protein test was positive (4+). Taking into consideration the patient's history, tuberculous abscess was considered, and tuberculous pleurisy was diagnosed as the cause of abnormal radioactive uptake in the left pleura. As the patient and his family did not consent to an open biopsy, treatment was initiated based on the diagnosis of pulmonary and thoracic TB of the left chest wall, with tuberculous pleurisy. The patient received $0.3 \mathrm{~g}$ isoniazid i.v.gtt qd, $0.45 \mathrm{~g}$ rifampincin i.v.gtt qd, $0.4 \mathrm{~g}$ amikacin i.v.gtt qd, $8.0 \mathrm{~g}$ aminosalicylic acid i.v.gtt qd, $0.4 \mathrm{~g}$ galtixacin p.o. qd, $0.75 \mathrm{~g}$ ethambutol hydrochloride p.o. qd, and $0.5 \mathrm{~g}$ pyrazinamide p.o. tid. Following anti-tuberculous treatment for 1 month, no significant change was observed in the lesion of the left chest wall. Thoracoscopic resection of the lump was performed on June 18, 2014, followed by histopathological 

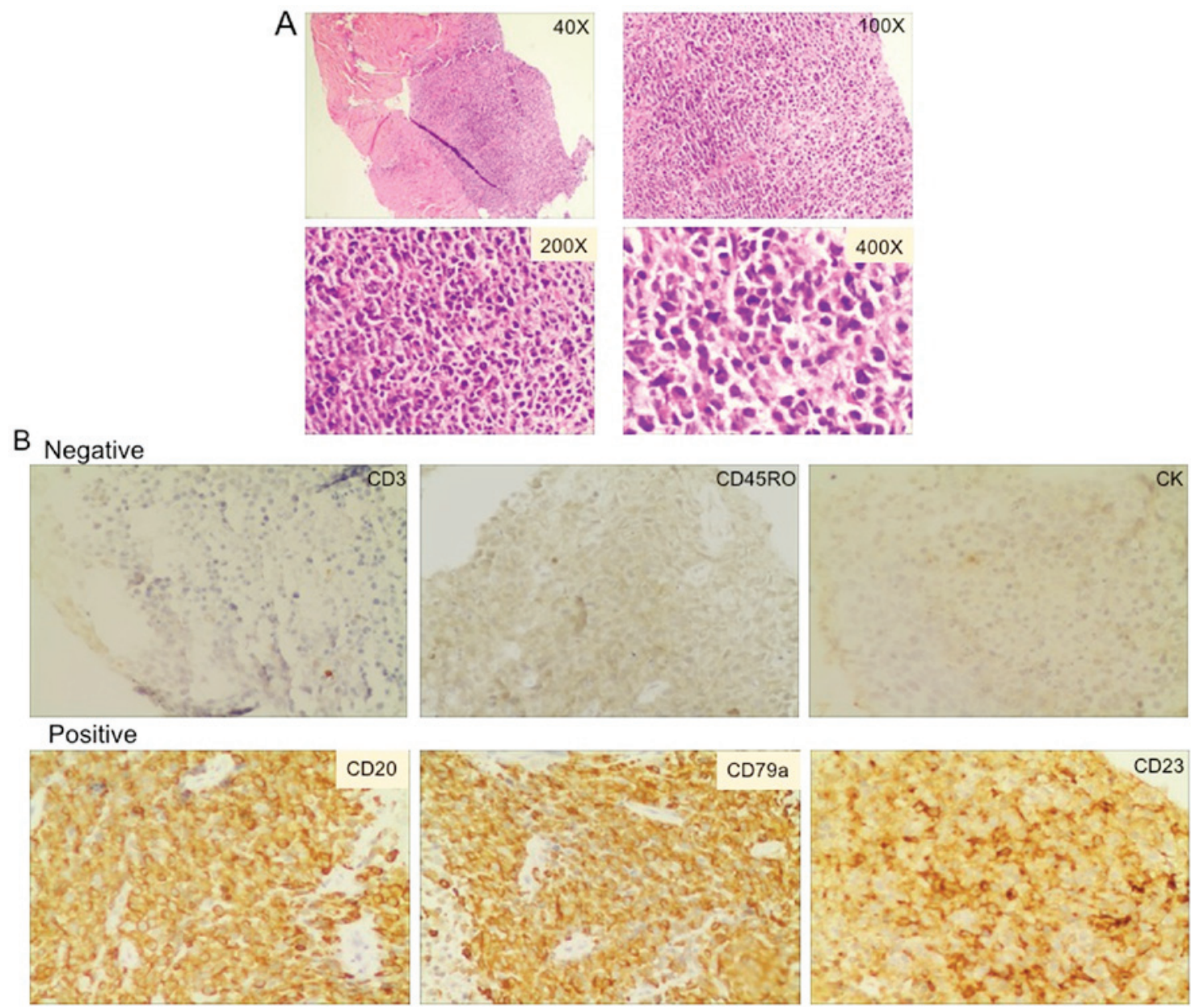

Figure 1. (A) Histopathology of the thoracoscopic biopsy specimen revealed confluent aggregates of numerous lymphocytes, exhibiting lack of germinal centers (hematoxylin and eosin staining; magnification, x40, x100, x200 and x400). Briefly, following deparaffinization and rehydration, the slides were stained with hematoxylin for $1.5 \mathrm{~min}$ at room temperature, rinsed with deionized water and dipped in tap water for 5 min to allow the stain to develop, then dipped in acid ethanol to destain. After rinsing with deionized water 3 times, the slides were stained with eosin for 3-4 sec at room temperature, followed by graded rehydration in 75,90 and 100\% ethanol. The slides were covered by coverslips with mounting medium and subjected to microscopic observation (B) Immunohistochemistry of the thoracoscopic biopsy specimen revealed positive CD20 (cat. no. ab78237, 1:200), CD79a (cat. no. ab79414, 1:100) and CD23 (cat. no. ab92495, 1:100) expression, and negative CD3 (cat. no. ab52959, 1:200), CD45RO (cat. no. ab23, 1:100) and CK (cat. no. ab82254, 1:100) expression on the surface of the cancer cells. All antibodies were obtained from Abcam, Cambridge, UK. CK, cytokeratin.

examination and consultation with the Fudan University Shanghai Cancer Center. At that time, the suspicion of diffuse large B-cell lymphoma (DLBCL) in the chest was raised. The examination of hematoxylin and eosin (H\&E)-stained sections (Fig. 1A) revealed a diffuse lymphoid infiltrate in the thoracic lump. Examination under high-power magnification revealed numerous large atypical lymphoid cells with irregular nuclei, which were double in size compared to a typical lymphocyte, with uneven chromatin distribution. The results of immunohistochemistry were as follows: CD20 ${ }^{+}, \mathrm{CD}^{-}$, B-cell lymphoma (Bcl)-6- ${ }^{-}$Bcl-2 $2^{+}, \mathrm{MUM1}^{+}, \mathrm{CD} 30^{+}$(diffuse and weak positivity), Epstein-Barr virus latent membrane protein $1^{+-}$(spotty positivity with nuclear localization), CD3-, AE1/AE3 ${ }^{-}$and $\mathrm{Ki}^{-} 67^{+}$ ( $95 \%$ ). Representative results of H\&E staining and immunohistochemistry are shown in Fig. 1.

On July 25, 2014, a positron emission tomography/CT scan (Fig. 2A), performed at Fudan University Shanghai Cancer Center revealed the following: i) A space-occupying lesion was identified on the left chest wall that invaded the neighboring ribs. An extremely high intensity of fluorine-18-fluorodeoxyglucose uptake was considered to indicate a possible lymphoma.
Multiple lymphatic invasions were demonstrated in the left axillary, internal mammary and intercostal lymph nodes and pleural effusion was observed in the left pleural space. ii) Left-sided pulmonary infection and hydropneumothorax were demonstrated. Old pathological changes were also observed in the left pleura. A subsequent bone marrow biopsy was negative for lymphoma. On August 9, 2014, a repeat thoracic CT (Fig. 2B), revealed that the lesion in the chest wall had significantly increased in size, causing destruction of the ribs. Furthermore, the left axillary nodes were found to be enlarged. On August 20, 2014, the patient was initiated on CHOP chemotherapy (cyclophosphamide $0.8 \mathrm{mg} / \mathrm{dl}$, doxorubicin hydrochloride $60 \mathrm{mg} / \mathrm{dl}$, with the addition of vincristine sulfate $2 \mathrm{mg} / \mathrm{dl}$, and prednisone $100 \mathrm{mg} / \mathrm{dl}$ ) for 8 cycles. Rituximab was not included in the regimen due to financial difficulties. Between the treatment cycles the status of the tumor was monitored by periodic thoracic CT scans [September 28 (Fig. 2C), October 21 and November 28 (Fig. 2D)], which revealed that the lesion had slightly shrunk. The therapeutic efficacy of CHOP chemotherapy was classified as stable disease. Overall, the treatment prolonged the patient's progression-free survival to $\sim 2$ months, and the overall survival 

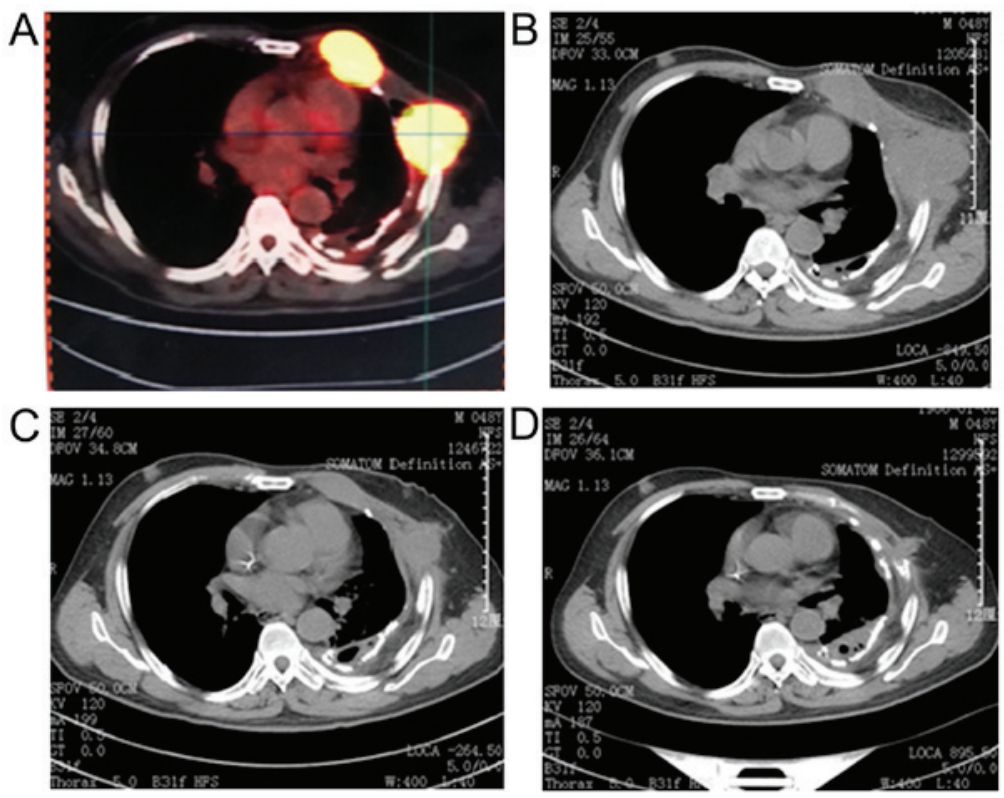

Figure 2. (A) Positron emission tomography/CT scan prior to chemotherapy. Space-occupying lesions were visible in the left chest wall with extremely high fluorodeoxyglucose metabolism, invading the ribs. (B) A repeat thoracic CT revealed an increase in the size of the lesions in the chest wall, with destruction of the neighboring ribs. The therapeutic efficacy of CHOP chemotherapy was monitored by thoracic CT on (C) September 28, 2014 and (D) November 28, 2014, which revealed that the lesion in the chest wall had shrunk slightly, and was evaluated as stable disease. CT, computed tomography.

to $\sim 1$ year. The patient ultimately succumbed to severe thoracic infection and pleural effusion.

\section{Discussion}

Lymphoma is a type of hematological malignancy that usually originates from lymph nodes or the lymphatic system. The two main types of lymphoma are NHL ( $90 \%$ of cases) and Hodgkin's lymphoma ( $10 \%$ of cases). NHL may be further subdivided into T-cell and B-cell lymphoma, with relatively rare cases of natural killer-cell lymphoma. DLBCL is the most common type of NHL $(1,5,6)$. In the majority of the cases, early-stage lymphoma is not associated with any specific clinical characteristics. Approximately $30-40 \%$ of the patients exhibit only general symptoms, such as fever, weight loss and night sweats. Physical signs of lymphoma include painless enlarged lymph nodes, enlarged spleen and local lumps (5). It has been reported that $25 \%$ of patients with NHL develop pleural effusion during disease progression $(7,8)$. Pathological changes in the lung have been demonstrated in $~ 50 \%$ of lymphoma patients on autopsy, the majority of which were metastases from mediastinal or pulmonary hilar lymph nodes $(7,9)$.

The risk of misdiagnosis is relatively high when lymphoma is complicated by pleural effusion, and it is often misdiagnosed as tuberculous pleurisy due to the high ADA level in the patient's pleural fluid, together with the clinical signs. ADA is considered as one of the key enzymes involved in purine metabolism; its primary function is associated with the development and maintenance of the immune system, as it plays an important role in T-cell differentiation and proliferation $(10,11)$. T cells mediating anti-TB immunity may cause ADA elevation in tuberculous pleural effusion (11). However, the invasion of the pleura by lymphoma also leads to a higher number of lymphocytes in the pleural effusion, increasing ADA concentration (10). Therefore, it may not be appropriate to set a high ADA level as the index for tuberculous pleurisy. It was reported recently by several studies that increased ADA level in pleural effusion may be an indication of lymphoma (10-12). According to Porcel et al, an extremely high ADA activity $(>250 \mathrm{U} / \mathrm{L})$ in the pleural fluid should raise the suspicion of empyema or lymphoma rather than TB (12).

In previous case reports on misdiagnosis of lymphoma, the majority of the cases were misdiagnosed as TB (9). The major causes of misdiagnosis are as follows. i) Lymphoma and TB share a number of common clinical characteristics, such as fever, night sweats, feeling of satiety after ingestion of only a small amount of food, fatigue and unexplained weight loss. ii) Particularly in China, the incidence of TB is significantly higher compared with that of lymphoma, and TB is usually preferentially considered, as clinicians are prone to suspect the most common and frequent disease. iii) Both lymphoma and TB originate at sites where a biopsy is generally difficult to perform. The patients and their families usually decline invasive examinations. iv) Clinicians may rely excessively on the results of biochemical and imaging examination for diagnosis, whereas they must improve their ability to perform a comprehensive analysis of the symptoms, clinical signs, bacteriology, histopathology and therapeutic efficacy.

In the present case, the causes of misdiagnosis may be summarized as follows. i) The patient was a middle-aged man who presented with a lump in the left chest wall, raising the suspicion of TB. ii) The radiographic changes observed on the thoracic CT scan matched left pulmonary TB and tuberculous pleurisy. iii) The biochemical analysis of the patient's pleural fluid revealed markedly elevated ADA levels (401.20 IU/L), which is characteristic of tuberculous pleurisy. iv) As the patient and his family did not consent to an open biopsy at first, pathological examination was delayed, as were diagnosis and treatment. 
There are several clinically important prognostic factors in NHL, including age, serum lactate dehydrogenase level, performance status, Ann Arbor stage and number of extranodal sites. When detected early, there is a better chance for quick diagnosis, timely treatment and improved overall survival. The standard treatment for DLBCL is chemotherapy in combination with immunotherapy $(13,14)$. The most common chemotherapy regimen is CHOP (cyclophosphamide, doxorubicin, vincristine and prednisone) plus rituximab for $\mathrm{CD}_{2} 0^{+}$patients. Additionally, autologous hematopoietic stem cell transplantation remains a treatment of choice for DLBCL patients with chemotherapy-sensitive disease relapse $(15,16)$.

\section{Acknowledgements}

Not applicable.

\section{Funding}

The present study was supported by Key Discipline (Oncology) in Medical Sciences of Jiaxing City (grant no. 04-F-14), and the Science and Technology Project of Jiaxing City (grant no. 2016AY23041).

\section{Availability of data and materials}

Not applicable.

\section{Authors' contributions}

$\mathrm{XY}$ and XX analyzed and interpreted the patient data regarding the lymphoma and the infectious condition. BS, QZ and XY conducted the final diagnosis of lymphoma based on the immunohistochemical and PET-CT results. BS made substantial contributions to the acquisition of the complete medical charts of the patient for this case report. QZ conducted the literature search and summarization during the whole process. YZ was a major contributor in drafting the manuscript. The final version of the manuscript has been read and approved by all authors.

\section{Ethics approval and consent to participate}

The present study meets all applicable standards with regard to the ethics of experimentation and research integrity, and has been approved by the Ethics Committee of the First Hospital of Jiaxing.

\section{Consent for publication}

Written informed consent has been obtained from the patient and his family for the publication of the case details and associated images.

\section{Competing interests}

The authors declare that they have no competing interests.

\section{References}

1. Armitage JO and Weisenburger DD: New approach to classifying non-Hodgkin's lymphomas: Clinical features of the major histologic subtypes. Non-Hodgkin's Lymphoma Classification Project. J Clin Oncol 16: 2780-2795, 1998.

2. Usuda D, Arahata M, Takeshima K, Sangen R, Takamura A, Kawai Y, Kasamaki Y, Iinuma Y and Kanda T: A case of diffuse large B-cell lymphoma mimicking primary effusion lymphoma-like lymphoma. Case Rep Oncol 10: 1013-1022, 2017.

3. Iwasa Y, Okada A, Takenaka H, Takahashi T, Koguchi N, Katayama K, Murakami S, Choh S, Tomoda K and Kimura H: Primary malignant lymphoma originating from the chest wall without preceding pleural disease. Intern Med 56: 681-686, 2017.

4. Krol EM, Ogrodnik A, Sieber S, Chronakos J and Mahfoozi AP: Unusual case of isolated pleural B-cell lymphoma. Conn Med 79: 347-349, 2015.

5. Abramson JS and Shipp MA: Advances in the biology and therapy of diffuse large B-cell lymphoma: Moving toward a molecularly targeted approach. Blood 106: 1164-1174, 2005.

6. Nogai H, Dörken B and Lenz G: Pathogenesis of non-Hodgkin's lymphoma. J Clin Oncol 29: 1803-1811, 2011.

7. Mian M, Wasle I, Gritsch S, Willenbacher W and Fiegl M: B cell lymphoma with lung involvement: What is it about? Acta Haematol 133: 221-225, 2015.

8. Vega F, Padula A, Valbuena JR, Stancu M, Jones D and Medeiros LJ: Lymphomas involving the pleura: A clinicopathologic study of 34 cases diagnosed by pleural biopsy. Arch Pathol Lab Med 130: 1497-1502, 2006.

9. Sun J, Li G, Zhang N, Li S and Chen R: Analysis of lymphoma presenting with pulmonary symptoms: Report of 79 cases. Zhonghua Jie He He Hu Xi Za Zhi 37: 597-600, 2014 (In Chinese).

10. Yao CW, Wu BR, Huang KY and Chen HJ: Adenosine deaminase activity in pleural effusions of lymphoma patients. QJM 107: 887-893, 2014.

11. Krenke R and Korczyński P: Use of pleural fluid levels of adenosine deaminase and interferon gamma in the diagnosis of tuberculous pleuritis. Curr Opin Pulm Med 16: 367-375, 2010.

12. Porcel JM, Esquerda A and Bielsa S: Diagnostic performance of adenosine deaminase activity in pleural fluid: A single-center experience with over 2100 consecutive patients. Eur J Intern Med 21: 419-423, 2010.

13. Vose JM: Molecular pathogenesis in non-Hodgkin lymphoma: Implications for therapy. Transfus Apheresis Sci 49: 155-156, 2013.

14. Fang $\mathrm{C}, \mathrm{Xu} \mathrm{W}$ and Li JY: A systematic review and meta-analysis of rituximab-based immunochemotherapy for subtypes of diffuse large B cell lymphoma. Ann Hematol 89: 1107-1113, 2010.

15. Gisselbrecht $\mathrm{C}$ : Is there any role for transplantation in the rituximab era for diffuse large B-cell lymphoma? Hematology Am Soc Hematol Educ Program 2012: 410-416, 2012.

16. Stiff PJ, Unger JM, Cook JR, Constine LS, Couban S, Stewart DA, Shea TC, Porcu P, Winter JN, Kahl BS, et al: Autologous transplantation as consolidation for aggressive non-Hodgkin's lymphoma. N Engl J Med 369: 1681-1690, 2013.
This work is licensed under a Creative Commons Attribution-NonCommercial-NoDerivatives 4.0 International (CC BY-NC-ND 4.0) License. 